\title{
ON THE MINIMUM MODULUS OF A ROOT OF A POLYNOMIAL
}

DENNIS P. VYTHOULKAS

Landau showed $[1]^{1}$ that the equation $1+z+\alpha z^{m}=0, m>1$, has at least one root with the modulus $\leqq 2$ and that the equation

$$
1+z+\alpha z^{m}+\beta z^{n}=0 \quad(1<m<n)
$$

has at least one root with the modulus $\leqq 17 / 3$. He posed the problem whether the equation

$$
P_{K}(z)=1+z+\alpha_{1} z^{n_{1}}+\cdots+\alpha_{K-1} z^{n_{K-1}}=0
$$

has at least one root with a modulus not greater than a number $M(K)$, depending only on the number of terms $P_{K}(z)$ and not at all on the numbers $\alpha_{1}, \alpha_{2}, \cdots, \alpha_{K-1}, n_{1}, n_{2}, \cdots, n_{K-1}$.

This problem was solved by $\mathrm{P}$. Montel. Montel [2] in his paper, written in 1923, showed that the number $M(K)$ has the simple value $K$, and that whenever the root assumes this maximum value $K$ all the roots of the polynomial are equal to $-K$.

In this note we establish the following stronger result.

Theorem. The equation $P_{K}(z)=1+z+\alpha_{1} z^{n}+\cdots+\alpha_{K-1} z^{n}{ }_{K-1}=0$ $\left(2 \leqq n_{1}<n_{2}<n_{3}<\cdots<n_{K-1}\right), \quad \alpha_{i} \neq 0 \quad(i=1,2, \cdots, K-1)$ has at least one root within or on the circumference of a circle with the center $-\lambda / 2$ and radius $\lambda / 2$ where $\lambda=\left(n_{1} /\left(n_{1}-1\right)\right) \cdot\left(n_{2} /\left(n_{2}-1\right)\right) \cdots$ $\left(n_{K-1} /\left(n_{K-1}-1\right)\right)$.

Proof. To prove the theorem and simplify operations let us consider the equation $f(z)=1+z+\alpha z^{m}+\beta z^{n}+\gamma z^{p}=0(2 \leqq m<n<p)$. Putting $z=1 / \delta$ we have $\phi(\delta)=\delta^{p}+\delta^{p-1}+\alpha \delta^{p-m}+\beta \delta^{p-n}+\gamma=0$; the derivative may be written $\phi^{\prime}(\delta)=\delta^{p-n-1} \cdot \phi_{1}(\delta)$ where

$$
\phi_{1}(\delta)=p \cdot \delta^{n}+(p-1) \delta^{n-1}+\alpha(p-m) \delta^{n-m}+\beta(p-n) .
$$

Similarly we write $\phi_{1}^{\prime}(\delta)=\delta^{n-m-1} \cdot \phi_{2}(\delta)$ where $\phi_{2}(\delta)=p \cdot n \delta^{m}+(p-1)$ $\cdot(n-1) \delta^{m-1}+\alpha(p-m)(n-m)$. Similarly we write $\phi_{2}^{\prime}(\delta)=p \cdot m n \delta^{m-2}$ $\cdot(\delta+\theta)$ where $\theta=(p-1) / p \cdot(n-1) / n \cdot(m-1) / m$.

Let $\Pi(x)$ be the half-plane $R(\delta) \geqq x$. If $0>x>-\theta, \phi_{2}(\delta)$ must have at least one root not in $\Pi(x)$, since otherwise by Lucas' Theorem [3] all the roots of $\phi_{2}^{\prime}(\delta)$ would be in $\Pi(x)$, and this contradicts the fact that $\delta=-\theta$ is a root of $\phi_{2}(\delta)$. Similarly $\phi_{1}(\delta)$ must have at least one

Presented to the International Congress of Mathematicians, September 1, 1950; received by the editors August 21, 1952 and, in revised form, January 4, 1954.

1 Numbers in brackets refer to the list of references. 
root not in $\Pi(x)$, and hence $\phi(\delta)$ must have at least one root not in $\Pi(x)$. But $\phi(\delta)$ has only a finite number of roots, so that if all these roots were in $R(\delta)>-\theta$ one could select an $x>-\theta$ so that all the roots would be in $\Pi(x)$. Hence $\phi(\delta)$ has at least one root in $R(\delta) \leqq-\theta$, and therefore $f(z)$ has at least one root in the image of this half-plane under $1 / z$, namely the circle $|z+1 / 2 \theta| \leqq 1 / 2 \theta$. It is easy to see that in the general case $1 / \theta$ is replaced by $\lambda$ and this completes the proof of the theorem.

Since $\lambda / 2 \leqq K / 2$ where $K+1$ is the number of the terms of the equation

$$
P_{K}(z)=1+z+\alpha_{1} z^{n_{1}}+\cdots+\alpha_{K-1} z^{n_{K-1}}=0,
$$

and since the circle of centre $-\lambda / 2$ and radius $\lambda / 2$ is covered by the circle of center $-K / 2$ and radius $K / 2$, we have the theorem:

Theorem. The polynomial $P_{K}(z)=1+z+\alpha_{1} z^{n_{1}}+\cdots+\alpha_{K-1} z^{n_{K-1}}$ of $K+1$ terms has at least one root within or on the circumference of a circle of centre $-K / 2$ and radius $K / 2$.

Corollary. The equation $\alpha_{0}+\alpha_{1} z+\alpha_{2} z^{2}+\cdots+\alpha_{m} z^{m}=0$ has at least one root within or on the circumference of a circle of centre $-\left(\alpha_{0} / \alpha_{1}\right)$ $\cdot m / 2$ and radius $\left|\alpha_{0} / \alpha_{1}\right| \cdot m / 2$.

If in the proof of the main theorem, the plane $\Pi(x)$ is replaced by any closed half-plane containing the origin but not containing $-\theta$, the reasoning is still valid and one obtains as a result the following:

THEOREM. Let $C$ be a closed circular disk containing on its boundary the points $z=0$ and $z=-\lambda$. Then $C$ contains at least one root of $P_{K}(z)$.

\section{REFERENCES}

1. E. Landau, Ueber den Picardschen Satz, Vierteljahrsschrift der Naturforschenden Gesellschaft in Zürich vol. 51 (1906) pp. 316-318. Sur quelque généralisation de theorème de M. Picard, Ann. École Norm. (3) vol. 24 (1907) pp. 179-201.

2. P. Montel, Sur les modules des zeros de polynomes, Ann. École Norm. vol. 40 (1923) p. 3.

3. Morris Marden, The geometry of the zeros of a polynomial in a complex variable, 1949, pp. 14-15.

Athens, Greece 